\title{
THE ZEROS OF LINEAR COMBINATIONS OF TRANSLATES OF POLYNOMIALS
}

\author{
PETER WALKER
}

(Received 26 September 2000; revised 7 February 2001)

\author{
Communicated by P. C. Fenton
}

\begin{abstract}
We investigate the location and separation of zeros of certain three-term linear combination of translates of polynomials. In particular, we find an interval of the form $I=[-1,1+h], h>0$ such that for a polynomial $f$, all of whose zeros are real, and $\lambda \in I$, all zeros of $f(x+2 i c)+2 \lambda f(x)+f(x-2 i c)$ are also real.
\end{abstract}

2000 Mathematics subject classification: primary $26 \mathrm{Cl} 10,30 \mathrm{C} 15$.

\section{Introduction}

Given the location of the zeros of a polynomial or entire function, it is important to have corresponding information about the zeros of other functions which may be derived from the given one. For instance Rolle's theorem says that if all the zeros of a polynomial lie on the real axis, then the zeros of the derivative also lie on the axis, and lie one in each interval between successive zeros. We showed in [3] that the separation, measured by

$$
\delta(f):=\min _{j}\left(a_{j}-a_{j-1}\right), \quad \text { where } f(x)=\prod_{j=1}^{n}\left(x-a_{j}\right), a_{1}<a_{2}<\cdots<a_{n},
$$

is increased by differentiation, and more generally in [4] that for real $k$ there is some explicit constant $c_{n}(k)>1$, for which

$$
\delta\left(f^{\prime}-k f\right) \geq c_{n}(k) \delta(f) .
$$

(C) 2002 Australian Mathematical Society $0263-6115 / 2002 \$ A 2.00+0.00$ 
Another operation which has been considered in this context is translation. For instance Pólya showed in [2, Lemma II, page 316], that for $c>0$ and $\theta \in C,|\theta|=1$, $f_{\theta}(x):=f(x+i c)+\theta f(x-i c)$ has only real zeros when $f$ does, and we showed in [5] that the separation is also increased:

$$
\delta\left(f_{\theta}\right)>\delta(f),
$$

again with explicit estimates for the amount of the increase. If we apply this result twice, using $\theta, \bar{\theta}=e^{ \pm i \alpha}, \alpha \in \mathbb{R}$, we find that when $f$ has only real zeros then the same is true for $f(x+2 i c)+2 \cos \alpha f(x)+f(x-2 i c)$. Equivalently the map

$$
f(x) \rightarrow T_{\lambda} f(x):=f(x+2 i c)+2 \lambda f(x)+f(x-2 i c),
$$

where $-1 \leq \lambda \leq 1, c>0$, preserves the reality of the zeros of $f$ and increases their separation. For convenience we shall replace $2 c$ by $c$ in (1) from now on.

In this paper we extend the range of these results to values of $\lambda$ which lie outside the interval $[-1,1]$. The motivation for doing this comes from the study of Mellin transforms in which given a function

$$
F(x)=\int_{0}^{\infty} t^{i x} \phi(t) d t
$$

we are interested in applying multipliers of the form $\left(t+r^{2} / t\right)$ and $\left(t+1 / r^{2} t\right)$ with real $r$ to the integrand. This gives

$$
\begin{aligned}
\int_{0}^{\infty} t^{i x}\left(t+r^{2} / t\right)\left(t+1 / r^{2} t\right) \phi(t) d t & =F(x+2 i)+\left(r^{2}+r^{-2}\right) F(x)+F(x-2 i) \\
& =F(x+2 i)+2 \lambda F(x)+F(x-2 i),
\end{aligned}
$$

where necessarily $\lambda>1$.

More precisely we shall find neighbourhoods of 1 and $\infty$ such that for these values of $\lambda$, all the zeros of $T_{\lambda}$ are real. (It is not possible to do this near -1 since two zeros are lost at $-\infty$.) The size of the neighbourhood of 1 turns out to depend only on the degree of the polynomial, and not on the values of $c$ or $\delta$, as Theorem 5 shows. We shall also estimate the separation of the zeros for $\lambda>1$.

Our results also relate to Pólya's generalisation of the Hermite-Poulain theoremsee [1], particularly Lemma I*, page 228 . This lemma states that if $\phi$ is in the Laguerre-Pólya class $L P$, and $g$ is a polynomial with real coefficients, then, with $D$ denoting the operation of differentiation, $\phi(D) g$ has at least as many real zeros as does $g$. In our context we have $T_{\lambda} f=2(\lambda+\cos (c D)) f$, and so $\phi(z)=\lambda+\cos (c z) \in L P$ if and only if $-1 \leq \lambda \leq 1$. In contrast, our Theorem 5 states that for suitable $f, T_{\lambda}$ also preserves the zeros of $f$ in some neighbourhood to the right of 1 . In addition, our Theorem 7 constructs, for any given $\lambda>1$, a polynomial with real zeros such that $T_{\lambda} f$ has some non-real zeros; this gives further information about the size of the neighbourhood of 1 , and shows that the restriction on $f$ cannot be omitted entirely. 


\section{Location of zeros}

Let $f(x):=\prod_{1}^{n}\left(x-a_{j}\right)$, where $a_{1}<a_{2}<\cdots<a_{n} \in \mathbb{R}$. For given $c>0$ and $x \in \mathbb{R}$, let

$$
a_{j}+i c-x:=r_{j} e^{i \theta_{j}}, \quad r_{j}>0, \quad 0<\theta_{j}=\cot ^{-1}\left(\frac{a_{j}-x}{c}\right)<\pi .
$$

Note that (i) each $\theta_{j}$ is a continuous strictly increasing function of $x$ with $\theta_{j}(-\infty)=0$, $\theta_{j}\left(a_{j}\right)=\pi / 2, \theta_{j}(+\infty)=\pi$ and (ii) that $\pi>\theta_{1}>\theta_{2}>\cdots>\theta_{n}>0$. Then

$$
\begin{aligned}
T_{\lambda} f(x) & =f(x+i c)+2 \lambda f(x)+f(x-i c) \\
& =\prod_{1}^{n}\left(x+i c-a_{j}\right)+\prod_{1}^{n}\left(x-i c-a_{j}\right)+2 \lambda \prod_{1}^{n}\left(x-a_{j}\right) \\
& =\prod_{1}^{n}\left(-r_{j} e^{-i \theta_{j}}\right)+\prod_{1}^{n}\left(-r_{j} e^{i \theta_{j}}\right)+2 \lambda \prod_{1}^{n}\left(-r_{j} \cos \theta_{j}\right) \\
& =2 \prod_{1}^{n}\left(-r_{j}\right)\left[\cos \sum_{1}^{n} \theta_{j}+\lambda \prod_{1}^{n} \cos \theta_{j}\right] .
\end{aligned}
$$

The following result follows at once from this expression:

LEMMA 1. Real zeros of $T_{\lambda} f$ occurwhere $\phi_{1}+\lambda \phi_{2}=0$, where

$$
\phi_{1}(x):=\cos \sum_{1}^{n} \theta_{j}(x), \quad \phi_{2}(x):=\prod_{1}^{n} \cos \theta_{j}(x),
$$

and since $T_{\lambda} f$ has degree $n$ (or $n-2$ in case $\lambda=-1$ ) all zeros of $T_{\lambda} f$ will be real if $n($ or $n-2)$ real zeros can be identified.

Let $b_{j}, 1 \leq j \leq n$, be the points at which $\sum_{1}^{n} \theta_{i}=(j-1 / 2) \pi$, that is, the zeros of $\phi_{1}$. Note that $\phi_{1} \rightarrow 1$ as $x \rightarrow-\infty$ and $\phi_{1} \rightarrow(-1)^{n}$ as $x \rightarrow+\infty$. Note also that $b_{1}<a_{1}<a_{n}<b_{n}$, since for instance $\theta_{1}=\pi / 2$ at $a_{1}$ and so $\sum_{1}^{n} \theta_{j}=\pi / 2$ at some point less than $a_{1}$. Let $c_{1}, c_{2}, \ldots, c_{n-1}$ be the points at which $\sum_{1}^{n} \theta_{j}=\pi, 2 \pi, \ldots,(n-1) \pi$, that is, the successive maxima and minima of $\phi_{1}$. Let $c_{0}=-\infty, c_{n}=+\infty$. Note that $\phi_{1}$ is strictly monotone from each $\left(c_{j-1}, c_{j}\right)$ onto $(-1,1)$ since each $\theta_{j}$ is an increasing function of $x$. More detailed information on the relation between $b_{j}, c_{j}$ and the roots $a_{j}$ of $f$ is contained in Lemma 3 below.

For $\phi_{2}$, observe that $\phi_{2} \rightarrow 1$ as $x \rightarrow-\infty$, that $\phi_{2} \rightarrow(-1)^{n}$ as $x \rightarrow \infty$, that $\phi_{2}$ changes sign at each $a_{j}$, and that $\left|\phi_{2}\right|<1$ for all real $x$. Let $h_{j}=\sup \left\{\left|\phi_{2}(x)\right|: a_{j}<\right.$ $\left.x<a_{j+1}\right\}, 1 \leq j \leq n-1$ with $h_{j}=\left|\phi_{2}\left(d_{j}\right)\right|$. Also let $h_{0}=\phi_{2}\left(b_{1}\right), h_{n}=\left|\phi_{2}\left(b_{n}\right)\right|$. Note that $h_{0}, h_{1}, \ldots, h_{n}$ are all in the interval $(0,1)$. Let $h_{*}=\min _{0 \leq j \leq n} h_{j}, h^{*}=$ $\max _{0 \leq j \leq n} h_{j}$. 
LEMMA 2. $T_{\lambda} f$ has only real zeros if either (i) $-1 \leq \lambda \leq 1 / h^{*}$ or (ii) $|\lambda| \geq 1 / h_{*}$.

PROOF. (i) For $-1<\lambda<1$, observe that $\left|\lambda \phi_{2}\right|<\left|\phi_{2}\right|<1$ for all $x$. Hence since the graph of $\phi_{1}$ goes from -1 to 1 on each interval $\left(c_{j-1}, c_{j}\right), 1 \leq j \leq n$, it follows from the the intermediate value theorem that there is at least one root of $\phi_{1}+\lambda \phi_{2}$ in each of these intervals. Since there are $n$ such intervals and at most $n$ zeros, the result follows in this case. If $\lambda \rightarrow-1$ the result remains true since the roots vary continuously with $\lambda$, though the two extreme zeros tend to $\pm \infty$.

For positive $\lambda, \phi_{1}$ and $-\lambda \phi_{2}$ have opposite sign outside $\left[b_{1}, b_{n}\right]$ (recall the earlier observation that $b_{1}<a_{1}<a_{n}<b_{n}$.) For $x \in\left[b_{1}, b_{n}\right]$ and $1 \leq \lambda<1 / h^{*}$ we still have $\left|\lambda \phi_{2}(x)\right|<1$ and hence there is exactly one root in each interval $\left(c_{j-1}, c_{j}\right)$ as before. The result when $\lambda=1 / h^{*}$ again follows as a limiting case.

(ii) If $|\lambda|>1 / h_{*}$, then we reverse the roles of $\phi_{1}, \lambda \phi_{2}$ in the above argument. The function $\lambda \phi_{2}$ now goes from a value less than -1 to a value greater than 1 on each interval $\left(d_{j-1}, d_{j}\right)$ (with $d_{0}=b_{1}$ and $d_{n}=b_{n}$ ), and so must intersect $\phi_{1}$ there. Since there are $n$ such intervals we have located the required number of real roots. The result when $|\lambda|=1 / h_{*}$ follows once more as a limiting case.

Lemma 2 shows the existence of some range of values of $\lambda>1$ for which the zeros

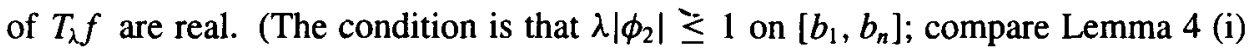
below.) However as it stands, it does not give useful quantitative information, since estimation of $h_{j}$ may involve the lengths of any of the intervals $\left(a_{j}, a_{j+1}\right)$. To make further progress we need more detailed information about the location of zeros of $T_{\lambda} f$ for which the following definition is required:

DEFINITION. For each $j, 1 \leq j \leq n$, let $I_{j}:=\left[a_{j}-\mu_{j}, a_{j}+v_{j}\right]$, where $\mu_{j}, v_{j}$ are non-negative numbers, which are defined by the requirement that the angles $\theta_{j}$ to the right of $a_{j}$ sum to $\pi / 2$ at $a_{j}-\mu_{j}$, and the complementary angles $\pi-\theta_{j}$ to the left of $a_{j}$ sum to $\pi / 2$ at $a_{j}+v_{j}$. More formally, we require that

$$
\sum_{k=j}^{n} \theta_{k}=\frac{\pi}{2}, \text { at } x=a_{j}-\mu_{j} \quad \text { and } \quad \sum_{k=1}^{j}\left(\pi-\theta_{k}\right)=\frac{\pi}{2}, \text { at } x=a_{j}+v_{j} .
$$

Since as has been noted, each $\theta_{k}$ is an increasing function of $x$ from $R$ onto $(0, \pi)$, and $\theta_{j}=\pi / 2$ at $a_{j}$, each of $\mu_{j}, v_{j}$ is well defined. (Note that in particular that $a_{1}-\mu_{1}=b_{1}, a_{n}+v_{n}=b_{n}$ and that $v_{1}=\mu_{n}=0$.) Let $I:=\bigcup_{1}^{n} I_{j}$.

The following result describes the arrangement of these intervals, and their relation to the points $b_{j}$ where $\phi_{1}=0$.

LEMMA 3. (i) The end points of the intervals $1_{j}$ form strictly increasing sequences:

$$
a_{j}-\mu_{j}<a_{j+1}-\mu_{j+1} \text { and } a_{j}+v_{j}<a_{j+1}+v_{j+1} \quad \text { for } 1 \leq j \leq n-1 \text {. }
$$


(ii) For each $j, 1 \leq j \leq n$, we have $b_{j} \in I_{j}$; more precisely

$$
\begin{aligned}
& a_{j}-\mu_{j} \leq b_{j}, \text { with strict inequality when } j>1, \text { and } \\
& a_{j}+v_{j} \geq b_{j}, \text { with strict inequality when } j<n .
\end{aligned}
$$

Moreover, if for some $k, a_{k} \leq b_{j} \leq a_{k+1}$, then $b_{j} \in I_{k} \cup I_{k+1}$. (This includes both of the extreme cases $k=0$, and $k=n$; if $k=0$ there is no $a_{k}$ to the left of $b_{j}$ and the claim is simply that $b_{j} \in I_{1}=\left[b_{1}, a_{1}\right]$, similarly if $k=n$, then $b_{j} \in I_{n}=\left[a_{n}, b_{n}\right]$.)

(iii) At any point not in $I, \phi_{1}$ and $\phi_{2}$ have strictly the same sign.

PROOF. (i) follows since each $\theta_{j}>0$ and $\sum_{j}^{n} \theta_{i}=\pi / 2$ at $a_{j}-\mu_{j}$ while $\sum_{j+1}^{n} \theta_{i}=$ $\pi / 2$ at $a_{j+1}-\mu_{j+1}$.

For (ii) we see that $\sum_{1}^{n} \theta_{i}=\pi / 2$ at $b_{1}$ and at $a_{1}-\mu_{1}$, so $b_{1}=a_{1}-\mu_{1}$ as already noted. In general, for $j \geq 2$ we have $\sum_{j}^{n} \theta_{i}=\pi / 2$ at $a_{j}-\mu_{j}$ and so $\sum_{1}^{n} \theta_{i}=\pi / 2+\sum_{1}^{j-1} \theta_{i}<\pi / 2+(j-1) \pi$. But $\sum_{1}^{n} \theta_{i}=(j-1 / 2) \pi$ at $b_{j}$, so $a_{j}-\mu_{j}<b_{j}$. The inequality $a_{j}+v_{j} \geq b_{j}$ is proved similarly, and so $b_{j} \in I_{j}$.

If $a_{k} \leq b_{j} \leq a_{k+1}$, then either $j \leq k$, when since $a_{j}+v_{j} \leq a_{k}+v_{k}$ and $b_{j} \in I_{j}$ we have also $b_{j} \in I_{k}$, or $j \geq k+1$ when $b_{j} \in I_{k+1}$ follows similarly.

(iii) For $x \notin I$, suppose that $a_{k}<x<a_{k+1}$ (which includes the extreme cases mentioned in the statement of the lemma). Since $x \notin I$, we must have $a_{k}+v_{k}<x<$ $a_{k+1}-\mu_{k+1}$. Then from the definition of $\nu_{k}, \mu_{k+1}$ we see that at $x$,

$$
0<p:=\sum_{i=k+1}^{n} \theta_{i}<\pi / 2 \text { and } 0<q:=\sum_{i=1}^{k}\left(\pi-\theta_{i}\right)<\pi / 2 .
$$

Hence $\sum_{i=1}^{n} \theta_{i}=k \pi+(p-q)$ must lie strictly between $(k-1 / 2) \pi$ and $(k+1 / 2) \pi$ and so $\phi_{1}(x)=\cos \left(\sum_{i=1}^{n} \theta_{i}\right)$ has the sign $(-1)^{k}$. But this is also the sign of $\phi_{2}$ on the interval $\left(a_{k}, a_{k+1}\right)$, so $\phi_{1}$ and $\phi_{2}$ have the same sign.

LEMMA 4. (i) If $0 \leq \lambda\left|\phi_{2}\right| \leq 1$ on $I$, then all zeros $\left(p_{j}\right)_{1}^{n}$ of $T_{\lambda} f$ are real and lie in $I$.

(ii) For each zero $p_{j}$ of $T_{\lambda} f$ there is some $a_{k}$ with

$$
\left|a_{k}-p_{j}\right| \leq 2 c \max (k, n+1-k) / \pi .
$$

(iii) $\sup \left\{\left|\phi_{2}(x)\right|: x \in I\right\} \leq 1 / \sqrt{1+\pi^{2} / 4 n^{2}}$.

ProOF. (i) We know from Lemma 2 that there is one zero $p_{j}$ in each interval $\left(c_{j-1}, c_{j}\right)$ and we shall assume without loss of generality that $\phi_{1}$ is increasing on this interval. Suppose that $a_{k} \leq b_{j} \leq a_{k+1}$. (including, as above, the extreme cases when $k=0$ or $k=n$ ). We consider separately the two cases when (a) $I_{k} \cap I_{k+1} \neq \emptyset$, and (b) $I_{k} \cap I_{k+1}=\emptyset$. 
In case (a) we have $\left[a_{k}, a_{k+1}\right] \subset I_{k} \cup I_{k+1}$, and suppose first that $c_{j-1} \leq a_{k}$. Then $\phi_{1}\left(a_{k}\right) \leq \phi_{1}\left(b_{j}\right)=0=-\lambda \phi_{2}\left(a_{k}\right)$. Otherwise if $c_{j-1} \geq a_{k}$, then $\phi_{1}\left(c_{j-1}\right)=$ $-1 \leq-\lambda \phi_{2}\left(c_{j-1}\right)$, since $c_{j-1}$ is now in $I_{k}$. Hence if $r:=\max \left(a_{k}, c_{j-1}\right)$, then $\phi_{1}(r) \leq-\lambda \phi_{2}(r)$. Similarly if $s:=\min \left(a_{k+1}, c_{j}\right)$, then $\phi_{1}(s) \geq-\lambda \phi_{2}(s)$. Hence from the intermediate value theorem, there is a zero of $\phi_{1}=-\lambda \phi_{2}$ on $[r, s] \subset\left[a_{k}, a_{k+1}\right] \subset I$ as required.

In case (b) when $I_{k} \cap I_{k+1}=\emptyset$ we can assume without loss of generality that $b_{j} \in I_{k}$. Now if $c_{j} \leq a_{k}+v_{k}$ then we can argue as in case (a) that there is a zero in $\left[r, c_{j}\right] \subset I_{k}$, where $r:=\max \left(a_{k}, c_{j-1}\right)$. But if $c_{j}>a_{k}+v_{k}$, then we know from Lemma 3 (iii) that $\phi_{1},-\phi_{2}$ have opposite signs off $I$, and so $\phi_{1}\left(a_{k}+v_{k}\right) \geq 0 \geq-\lambda \phi_{2}\left(a_{k}+v_{k}\right)$ and there is a zero in $\left[r, a_{k}+v_{k}\right] \subset I_{k}$. This proves (i).

(ii) We have shown in (i) that each $p_{j}$ lies in some $I_{k}$, so it is enough to prove the inequalities

$$
\mu_{j}<2 c(n-j+1) / \pi, \quad \nu_{j}<2 c j / \pi .
$$

But at $x=a_{j}-\mu_{j}$, we have

$$
\frac{\pi}{2}=\sum_{k=j}^{n} \theta_{k}<\sum_{k=j}^{n} \tan \theta_{k} \leq(n-j+1) \tan \theta_{j}=\frac{(n-j+1) c}{\mu_{j}}
$$

which gives the result for $\mu_{j}$, and the result for $\nu_{j}$ is similar.

(iii) For each point $x \in I$, we have $x \in I_{k}$ for some $k$ and so

$$
\left|x-a_{k}\right| \leq \max \left(\mu_{k}, v_{k}\right) \leq 2 c n / \pi .
$$

Thus at $x$ we have

$$
\left|\cos \theta_{k}\right|=\frac{\left|x-a_{k}\right|}{\sqrt{\left(x-a_{k}\right)^{2}+c^{2}}} \leq \frac{1}{\sqrt{1+\pi^{2} / 4 n^{2}}},
$$

and so

$$
\sup \left\{\left|\phi_{2}(x)\right|: x \in I\right\} \leq \frac{1}{\sqrt{1+\pi^{2} / 4 n^{2}}}
$$

We now come to our main result on the zeros of $T_{\lambda} f$.

THEOREM 5. If $1 \leq \lambda \leq \sqrt{1+\pi^{2} / 4 n^{2}}$, then $T_{\lambda} f$ has only real zeros whose separation satisfies

$$
\delta\left(T_{\lambda} f\right) \geq \frac{2 d}{\pi} \tanh \left(\frac{\pi c}{d}\right) \cos ^{-1}\left(\frac{\lambda}{\sqrt{1+\pi^{2} / 4 n^{2}}}\right)
$$

where $d=\delta(f)=\min _{j}\left(a_{j}-a_{j-1}\right)$. 
The reality of the zeros follows at once from Lemma 4 , since the restriction on $\lambda$ ensures that $\lambda\left|\phi_{2}\right| \leq 1$ on $I$. The proof of the estimate will follow after we have established Lemma 6 below.

The following example shows that there can be no corresponding result for infinite products, without further restrictions on the location of their zeros.

EXAMPLE. Let $f(x)=\prod_{1}^{\infty}\left\{(1-x / n) e^{x / n}\right\}$. Then $T_{\lambda} f$ has some non-real roots for any $\lambda>1$.

In this example we have $\theta_{n}=\cot ^{-1}\{(n-x) / c\}$ and we write $n \pm i c=x+r_{n} e^{ \pm i \theta_{n}}$ as in Lemma 1. Then

$$
\begin{aligned}
T_{\lambda} f(x)= & \prod_{1}^{\infty}\left\{\left(1-\frac{x+i c}{n}\right) e^{(x+i c) / n}\right\}+\prod_{1}^{\infty}\left\{\left(1-\frac{x-i c}{n}\right) e^{(x-i c) / n}\right\} \\
& +2 \lambda \prod_{1}^{\infty}\left\{\left(1-\frac{x}{n}\right) e^{x / n}\right\} \\
= & \prod_{1}^{\infty}\left\{\frac{r_{n}}{n} e^{x / n+i\left(c / n-\theta_{n}\right)}\right\}+\prod_{1}^{\infty}\left\{\frac{r_{n}}{n} e^{x / n-i\left(c / n-\theta_{n}\right)}\right\}+2 \lambda \prod_{1}^{\infty}\left\{\frac{r_{n}}{n} e^{x / n} \cos \theta_{n}\right\} \\
= & 2 \prod_{1}^{\infty}\left\{\frac{r_{n}}{n} e^{x / n}\right\}\left[\cos \left(\sum_{1}^{\infty}\left(\theta_{n}-\frac{c}{n}\right)\right)+\lambda \prod_{1}^{\infty} \cos \theta_{n}\right],
\end{aligned}
$$

where it is easily checked that the sums and products are absolutely convergent as grouped. This gives an obvious extension of Lemma 1 . We shall show that the sum $\sum_{1}^{\infty}\left(\theta_{n}-c / n\right) \rightarrow-\infty$ as $x \rightarrow-\infty$; this will establish the result since $\phi_{1}=\cos \left(\sum_{1}^{\infty}\left(\theta_{n}-c / n\right)\right)$ oscillates infinitely often between 1 and -1 , while $\phi_{2}=$ $\prod_{1}^{\infty} \cos \theta_{n} \rightarrow 1$ as $x \rightarrow-\infty$ since each term $\rightarrow 1$ and the product is absolutely convergent. Thus as soon as $\lambda>1$, some real intersections of $\phi_{1}$ and $-\lambda \phi_{2}$ will be replaced by corresponding non-real roots. It remains to show that $\sum_{1}^{\infty}\left(\theta_{n}-c / n\right) \rightarrow$ $-\infty$ as $x \rightarrow-\infty$.

Given $K>0$, choose $N \geq 3$ such that (i) $\sum_{N+1}^{\infty}\left(\theta_{n}-c / n\right)<1$ and (ii) $\sum_{1}^{N} c / n>$ $2 K$. Since each $\theta_{n} \rightarrow 0$ as $x \rightarrow-\infty$ we can choose $x$ sufficiently large and negative such that $\theta_{n}<1 / N$ for $n=1,2, \ldots, N$. Then for such $x$ we will have

$$
\sum_{1}^{\infty}\left(\theta_{n}-\frac{c}{n}\right)=\sum_{1}^{N} \theta_{n}-\sum_{1}^{N} \frac{c}{n}+\sum_{N+1}^{\infty}\left(\theta_{n}-\frac{c}{n}\right)<1-2 K+1
$$

which is less than $-K$ when $K>2$. This shows that $\sum_{1}^{\infty}\left(\theta_{n}-c / n\right) \rightarrow-\infty$ as $x \rightarrow-\infty$ as required.

The following estimate for the rate of increase of the sum of the angles $\theta_{j}$ is needed to complete the proof of Theorem 5 . 
LEMMA 6. For all $x \in \mathbb{R}$,

$$
\frac{d}{d x}\left(\sum_{j=1}^{n} \theta_{j}\right)<\frac{\pi}{d} \operatorname{coth}\left(\frac{\pi c}{d}\right),
$$

where $d=\delta(f)=\min \left(a_{j}-a_{j-1}\right)$.

PROOF. We have $\theta_{j}=\cot ^{-1}\left(\left(a_{j}-x\right) / c\right)$ and hence $\theta_{j}^{\prime}=c /\left(c^{2}+\left(a_{j}-x\right)^{2}\right)$. Let $h=\min _{j}\left|a_{j}-x\right|$. Since the zeros are separated by at least $d=\delta(f)$ the sum $\sum_{1}^{n} \theta_{j}^{\prime}$ is bounded above by

$$
\begin{aligned}
\sum_{j=-\infty}^{\infty} \frac{c}{c^{2}+(h+j d)^{2}} & =\frac{\pi}{d} \frac{\sinh (2 \pi c / d)}{\cosh (2 \pi c / d)-\cos (2 \pi h / d)} \\
& \leq \frac{\pi}{d} \frac{\sinh (2 \pi c / d)}{\cosh (2 \pi c / d)-1}=\frac{\pi}{d} \operatorname{coth}\left(\frac{\pi c}{d}\right)
\end{aligned}
$$

We can now complete the proof of Theorem 5 .

Proof. Put $\lambda_{0}=\sqrt{1+\pi^{2} / 4 n^{2}}$ and let $\sigma$ denote any real number in $\left(1, \lambda_{0}\right)$. Then for $x \in I$ and $1 \leq \lambda \leq \sigma$ we have from (2) that

$$
\lambda\left|\phi_{2}(x)\right| \leq \lambda / \lambda_{0} \leq \sigma / \lambda_{0}<1 .
$$

In particular, each zero $p_{j-1}$ of $\mathcal{T}_{\lambda} f$ lies in that part of the interval $\left(c_{j-1}, c_{j}\right)$ on which $\left|\phi_{1}(x)\right| \leq \sigma / \lambda_{0}$. It follows that between $\mathrm{p}_{j-1}$ and $c_{j}$ the sum $\sum \theta_{j}$ must increase by at least $\cos ^{-1}\left(\sigma / \lambda_{0}\right)$. Similarly between $c_{j}$ and $p_{j}$ the sum $\sum \theta_{j}$ must increase by at least $\cos ^{-1}\left(\sigma / \lambda_{0}\right)$, and so on $\left[p_{j-1}, p_{j}\right]$ the sum $\sum \theta_{j}$ must increase by at least $2 \cos ^{-1}\left(\sigma / \lambda_{0}\right)$.

But $\phi_{1}(x)=\cos \left(\sum \theta_{j}\right)$ and so $\left|\phi_{1}^{\prime}(x)\right| \leq \sum \theta_{j}^{\prime} \leq(\pi / d) \operatorname{coth}(\pi c / d)$ from Lemma 6. Hence an increase in $x$ of at least $\Delta$ is required to make $\sum \theta_{j}$ increase by $2 \cos ^{-1}\left(\sigma / \lambda_{0}\right)$ where

$$
\Delta \frac{\pi}{d} \operatorname{coth}\left(\frac{\pi c}{d}\right) \geq 2 \cos ^{-1}\left(\frac{\sigma}{\lambda_{0}}\right) .
$$

Hence the separation $\delta\left(T_{\lambda} f\right)$ is at least $(2 d / \pi) \tanh (\pi c / d) \cos ^{-1}\left(\sigma / \lambda_{0}\right)$ and putting $\lambda=\sigma$ gives the required result.

We complement Theorem 5 with the following result which shows that for $\lambda>1$, some restriction on $f$ is necessary to ensure reality of the zeros of $T_{\lambda} f$.

THEOREM 7. Given any $\lambda>1$, there is a polynomial $f$ with real distinct zeros such that not all zeros of $T_{\lambda} f$ are real. 
PROOF. Consider first $f(x)=x^{n}$, where all zeros are at the origin. With the notation of Lemma 1 , we have all $\theta_{j}=\theta:=\cot ^{-1}(x / c), 0<\theta<\pi$, and so the zeros of $T_{\lambda} f$ are where $\cos (n \theta)+\lambda(\cos \theta)^{n}=0$.

We see by inspection of the graphs of $\cos (n \theta)$ and $-\lambda(\cos \theta)^{n}$ that when $\lambda=1$ there are two real zeros on the interval $[\pi / 2 n, 3 \pi / 2 n]$. However if $\lambda$ is increased so that $-\lambda(\cos \theta)^{n}=-1$ at $\theta=3 \pi / 2 n$ then these real zeros are lost. Thus we have to show that for any $\lambda>1$ we can choose $n$ so that $\lambda(\cos (3 \pi / 2 n))^{n}>1$. This follows at once since $(\cos (3 \pi / 2 n))^{n} \rightarrow 1$ as $n \rightarrow \infty$, and we can estimate the size of $n$ from the elementary inequalities

$$
\left(\cos \left(\frac{3 \pi}{2 n}\right)\right)^{n}>\left(1-\frac{1}{2}\left(\frac{3 \pi}{2 n}\right)^{2}\right)^{n}>1-\frac{9 \pi^{2}}{8 n} .
$$

Thus given $\lambda>1$, we can say that not all zeros of $T_{\lambda}\left(x^{n}\right)$ are real if

$$
\lambda>\frac{1}{1-9 \pi^{2} / 8 n}, \quad \text { or equivalently } n>\frac{9 \pi^{2} \lambda}{8(\lambda-1)} .
$$

Finally to find an example with distinct roots, we can replace $x^{n}$ by a polynomial $n$ zeros equally spaced on $[-h, h]$ say, and take $h$ sufficiently close to zero.

It would be interesting to find which of the estimates $1+O\left(n^{-2}\right)$ given by Theorem 5 , or $1+O\left(n^{-1}\right)$ for the example of Theorem 7 is closer to the correct order of magnitude for the value of $\lambda$ required to make the zeros of $T_{\lambda} f$ real.

\section{References}

[1] G. Pólya, 'Algebraische Untersuchungen über ganze Funktionen vom Geschlechte Null und Eins', J. Reine Angew. Math. 145 (1915), 224-249.

[2] — , 'Bemerkung über die Integraldarstellung der Riemannsche $\xi$-Function', Acta Math. 48 (1926), 305-317.

[3] P. L. Walker, 'Separation of the zeros of polynomials', Amer. Math. Monthly 100 (1993), 272-273.

[4] _ , 'Bounds for the separation of real zeros of polynomials', J. Austral. Math. Soc. (Series A) 59 (1995), 330-342.

[5] _-, 'Separation of zeros of translates of polynomials and entire functions', J. Math. Anal. Appl. 206 (1997), 270-279.

College of Arts and Science

American University of Sharjah

P.O. 26666 Sharjah

United Arab Emirates

e-mail: peterw@aus.ac.ae 
